\title{
(6) OPEN ACCESS \\ Analysis of head impact exposure and brain microstructure response in a season-long application of a jugular vein compression collar: a prospective, neuroimaging investigation in American football
}

\author{
Gregory D Myer, ${ }^{1,2,3,4,5,6}$ Weihong Yuan, ${ }^{7,8}$ Kim D Barber Foss, 1,2,9,10 \\ Staci Thomas, ${ }^{1,2}$ David Smith, ${ }^{1}$ James Leach, ${ }^{11}$ Adam W Kiefer, ${ }^{1}, 2,3,12$ \\ Chris Dicesare, ${ }^{1,2}$ Janet Adams, ${ }^{11}$ Paul J Gubanich, ${ }^{1,3}$ Katie Kitchen, ${ }^{1,2}$ \\ Daniel K Schneider, ${ }^{1,2,13}$ Daniel Braswell, ${ }^{1,2}$ Darcy Krueger, ${ }^{14}$ Mekibib Altaye ${ }^{15}$
}

- Additional material is published online only. To view please visit the journal online (http://dx.doi.org/10.1136/ bjsports-2016-096134).

For numbered affiliations see end of article.

\section{Correspondence to} Dr Gregory D Myer, Cincinnati Children's Hospital, 3333 Burnet Avenue; MLC 10001 Cincinnati, OH 45229, USA: Greg.Myer@cchmc.org

Accepted 21 May 2016 Published Online First 15 June 2016
CrossMark

To cite: Myer GD, Yuan W, Barber Foss $\mathrm{KD}$, et al. $\mathrm{Br} \mathrm{J}$ Sports Med 2016;50:12761285.

\section{ABSTRACT}

Background Historical approaches to protect the brain from outside the skull (eg, helmets and mouthpieces) have been ineffective in reducing internal injury to the brain that arises from energy absorption during sportsrelated collisions. We aimed to evaluate the effects of a neck collar, which applies gentle bilateral jugular vein compression, resulting in cerebral venous engorgement to reduce head impact energy absorption during collision. Specifically, we investigated the effect of collar wearing during head impact exposure on brain microstructure integrity following a competitive high school American football season.

Methods A prospective longitudinal controlled trial was employed to evaluate the effects of collar wearing $(n=32)$ relative to controls (CTRL; $n=30$ ) during one competitive football season (age: $17.04 \pm 0.67$ years). Impact exposure was collected using helmet sensors and white matter (WM) integrity was quantified based on diffusion tensor imaging (DTI) serving as the primary outcome.

Results With similar overall g-forces and total head impact exposure experienced in the two study groups during the season ( $p>0.05)$, significant preseason to postseason changes in mean diffusivity, axial diffusivity and radial diffusivity in the WM integrity were noted in the CTRL group (corrected $p<0.05$ ) but not in the collar group ( $p>0.05$ ). The CTRL group demonstrated significantly larger preseason to postseason DTI change in multiple WM regions compared with the collar group (corrected $p<0.05$ ).

Discussion Reduced WM diffusivity alteration was noted in participants wearing a neck collar after a season of competitive football. Collar wearing may have provided a protective effect against brain microstructural changes after repetitive head impacts.

Trial registration number NCT02696200.

\section{INTRODUCTION}

Sports-related traumatic brain injury (TBI) is common in competitive sports and recreational activities, with 1.6-3.8 million cases reported annually in the USA. ${ }^{1}$ Children, adolescents and young adults are at the highest risk for sustaining mild TBI (mTBI). ${ }^{2}$ A recent review of personal protective equipment concluded that, while some items might confer a preventative effect from superficial head injury, they all failed to significantly prevent sports-related TBI in athletes. ${ }^{3}$ In American football specifically, prospective studies of mouthguards (both specialised and custom-fitted models) indicate that they may have minimal, if any, effect on sportsrelated TBI incidence. ${ }^{4-8}$ There is also limited highlevel prospective, objective evidence indicating that any recent helmet design can prevent sports-related TBI. Helmets were developed for, and are effective in, preventing skull fractures and intracranial hematomas- ${ }^{9}$ they do not however protect the brain inside the cranium. This lack of measureable protection may be due to the limited ability of helmets to minimise the collision of the brain against the inside of the skull (ie, slosh) and from differential acceleration between the skull and its contents. ${ }^{10}$

We evaluated physiological-based slosh mitigation (ie, reduced movement of fluids within moving containers) with increased elevation found to be associated with reduced sports-related TBI rates at higher altitudes. ${ }^{11} 12$ We postulated that acclimatisation to altitude may have influenced an increased intracranial blood volume, resulting in a tighter fit of the brain within the cranium. The proposed physiological response to decreased relative ambient oxygen (thus increasing intracranial flow and volume) was speculated to have protected the athletes at higher elevations against sports-related TBI. ${ }^{11} 12$

We also investigated mechanical-based slosh mitigation, in the form of a jugular vein compression collar fashioned off of the diagnostic Queckenstedt manoeuvre. ${ }^{13}$ This approach demonstrated an $83 \%$ reduction in amyloid precursor protein-positive axons-a widely accepted biomarker of TBIduring a $900 \mathrm{~g}$ impact protocol studied in animals. ${ }^{14}$ Further, an associated preclinical TBI model, which also used the same impact protocol, demonstrated a $>45 \%$ reduction in degenerative neurons, reactive astrocytes and microglial activation with the application of jugular vein impedance. ${ }^{15}$ The proposed mechanism of protection was achieved via the mechanical impedance of the internal jugular vein (IJV) which is proposed to result in increased cerebral venous sinus engorgement to promote a 'tighter fit' of the brain inside the cranium. ${ }^{14} 15$

Drawing from these studies, a lightweight neck collaring compressive device was developed for humans (figure 1). Much like the effect of collar in 


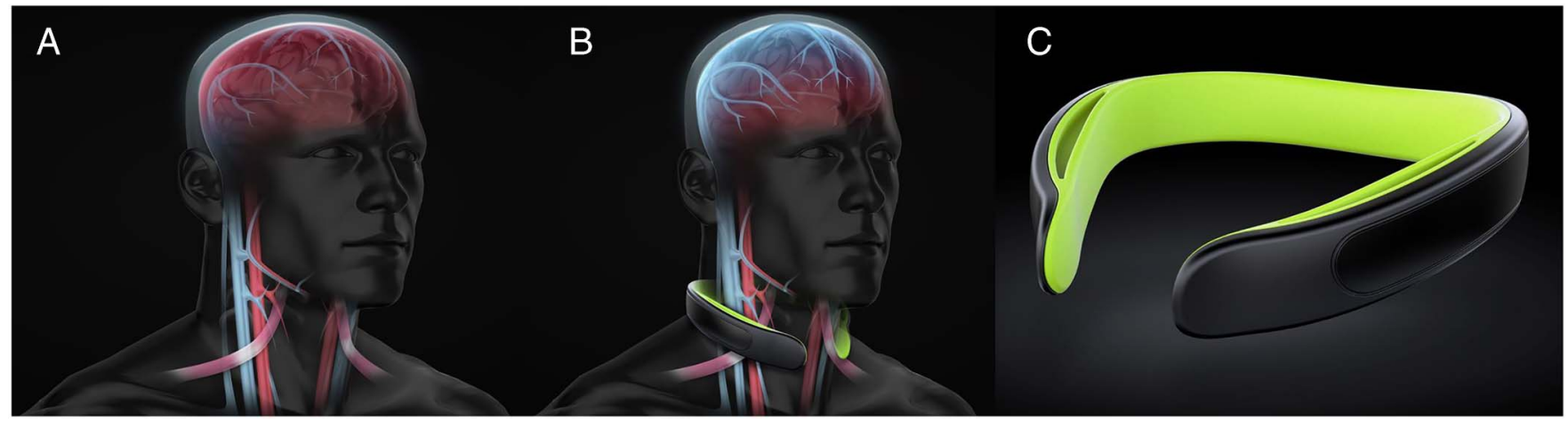

Figure 1 (A) Visual representation of the jugular vein and intracranial venous system. (B) Visual representation of the collar application located near the omohyoid process. The increased jugular vein dilation superior to the collar application and resultant backfilling of the intracranial venous system are represented to provide the hypothesised tighter fit of the brain inside the cranium. (C) Neck collar device employed in current investigation.

animal experiment, jugular vein compression in humans results in increased volume of the venous capacitance vessels of the cranium. ${ }^{16}$ Filling the compensatory reserve volume ${ }^{17}$ of the brain and spinal column appears to increase brain stiffness during jugular compression, as assessed by magnetic resonance elastography. ${ }^{18}$ Based on prior physiological models, we hypothesised that this would create a cradling effect to increase the brain's resistance to movement or inertia, (ie, slosh mitigation of the brain inside the skull), potentially protecting the brain from external head impacts. ${ }^{11}$ A preliminary prospective longitudinal investigation showed that alterations in white matter (WM) microstructure and electrophysiological measures were ameliorated by application of jugular compression during head impact exposure in hockey. ${ }^{19}$ Therefore, the purpose of the current investigation was to examine the effects of a jugular vein compression collar worn during a competitive American football season to determine the potential effect on neuroimaging biomarkers associated with brain injury. Changes in brain diffusivity (a measure of WM brain injury ${ }^{20}$ that has been reported in neuroimaging studies of sports-related TBI ${ }^{21}$ were evaluated in relation to the cumulative effects of repetitive head impacts accrued throughout the season. ${ }^{22}$

\section{METHOD}

\section{Study participants}

The Cincinnati Children's Hospital Medical Center Institutional Review Board (IRB) approved the data collection procedures and consent forms. The IRB approval number is IRB \# 2015-2205 (ClinicalTrials.gov \#: NCT02696200). Sixty-two healthy male varsity high school football players were enrolled from Southwest Ohio, USA. Parents, guardians and athletes provided informed consent prior to participation in the study. Primary exclusion criteria included history of neurological deficits, previous cerebral infarction, previous severe head trauma, known increased intracranial pressure, metabolic acidosis or alkalosis, glaucoma (narrow angle or normal tension), hydrocephalus, penetrating brain trauma (within 6 months), known carotid hypersensitivity, central vein thrombosis, known airway obstruction or seizure disorder. Teams were assigned to either the collar group or the control (CTRL) group prior to the start of the season. Of the 62 participants enrolled, 1 participant had contraindications for MRI (dental braces); 1 athlete had test anxiety with MR testing; 2 participants did not complete all testing due to medical issues that arose during the season unrelated to football participation; and 2 participants suffered a season-ending injury. Twelve participants had unusable MRI data on either preseason or postseason testing due to motion artefact during the scan and were excluded from all imaging analysis leaving 42 (21 collar and 21 CTRL) study participants (age $17.13 \pm 0.66$ years; figure 2 ). In addition, one athlete in the collar group did not have accelerometer covariate information and, therefore, was not included in the correlation analysis. No significant difference was found between the athletes excluded for imaging reason and those who remained for analysis in age and all the impact-related variables (number of hits, cumulative

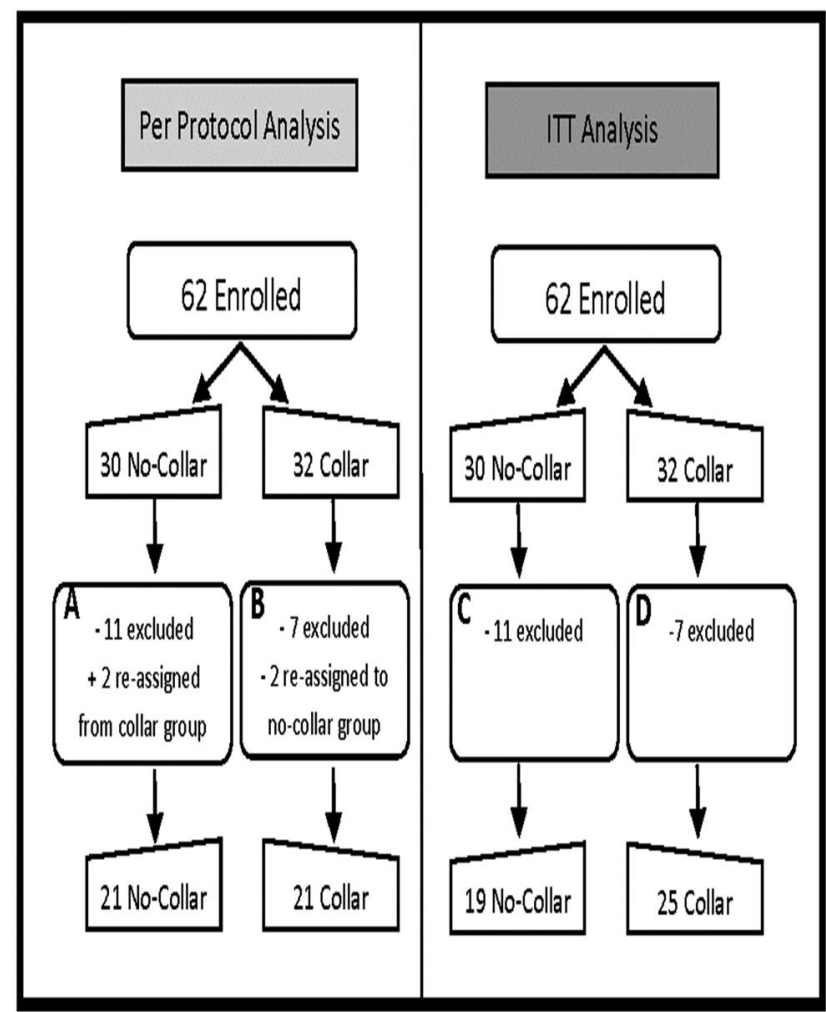

Figure 2 Study participant flow chart. (A) 11 excluded: no post-test (3), movement on MRI (7), season-ending injury (1); 2 reassigned from collar group due to non-compliance of collar wearing. (B) 7 excluded: movement on MRI (5), contraindication to MRI (1), season-ending injury (1). (C) 11 excluded: no post-test (3), movement on MRI (7), season-ending injury (1). (D) 7 excluded: movement on MRI (5), contraindication to MRI (1), season-ending injury (1). ITT, intention to treat. 
g-force, average g-force per hit) at all the g-force thresholds (all hits, and g-force $>20,50,100$ or $150 \mathrm{~g}$ ).

\section{Instrumentation and procedures}

Testing was completed at preseason and postseason. Each testing session consisted of a preimaging screening questionnaire to assess for safety of brain MRI. Preseason (baseline) testing took place prior to the start of the first practice, while postseason testing took place after the completion of competitive play (including postseason play, when applicable). The average time between testing was $129.7 \pm 14.9$ days (range 95-154 days). During the study period, 60 practices, 10 regular season games and 2 playoff games for the collar group and 50 practices, 10 regular season games and no playoff games for the CTRL group were completed. The postseason imaging data were acquired at $7.05 \pm 4.61$ and $5.83 \pm 6.70$ days $(p=0.50)$ after the last competitive event in the CTRL group and the collar group, respectively (figure 2).

\section{MRI data acquisition}

All MRI data were acquired on the same $3 \mathrm{~T}$ Philips Achieva MRI scanner (Philips Medical Systems, Best, the Netherlands) using a 32-channel head coil. The diffusion tensor imaging (DTI) data were acquired with a spin echo-planar imaging sequence with the following specifications: repetition time/echo time $(\mathrm{TR} / \mathrm{TE})=9000 / 83 \mathrm{~ms}$; field of view $(\mathrm{FOV})=256 \times 256 \mathrm{~mm}$; matrix $=128 \times 128$; in-plane resolution $=2 \times 2 \mathrm{~mm}$; slice thickness $=2 \mathrm{~mm} ; 72$ slices. Diffusion-weighted images were acquired along 61 non-collinear directions with 7 non-diffusionweighted images $\left(\mathrm{b} 0=1000 \mathrm{~s} / \mathrm{mm}^{2}\right)$. A high-resolution, threedimensional (3D) T1-weighted anatomical dataset was acquired in the sagittal direction with the following specifications: TR/ $\mathrm{TE}=8.1 / 3.7 \mathrm{~ms} ; \mathrm{TI}=1070 \mathrm{~ms} ; \mathrm{FOV}=256 \times 256 \mathrm{~mm}$; matrix $=$ $256 \times 256$; in-plane resolution $=1 \times 1 \mathrm{~mm}$; slice thickness $=1 \mathrm{~mm}$; 180 slices. Susceptibility-weighted imaging (SWI) was also performed (venous blood-oxygen-level dependent (BOLD), TR/ $\mathrm{TE}=14.7 / 20.7 \mathrm{~ms}, \quad \mathrm{FOV}=220 \times 220 \mathrm{~mm}, \quad$ flip angle $=10^{\circ}$, matrix $=220 \times 221$, slice thickness $=2 \mathrm{~mm}$ ). One board-certified neuroradiologist (JLL), blinded to treatment group assignment, evaluated all anatomical images (3D T1-weighted and SWI images). Clinically significant findings were reported to the study investigators, participants and parents as per study protocol. On subjective evaluation of any of the anatomical images (T1-weighted and SWI images), there were no traumatic abnormalities identified. There were no changes between the preseason and postseason anatomical images in any participant, and there was no evidence of any intracranial complication (eg, brain haemorrhage or lesion) from collar use.

DTI data were processed with the Functional MRI of the Brain (FMRIB) Software Library (FSL) software package (http:// www.fmrib.ox.ac.uk/fsl). In FSL, skull stripping was performed using the brain extraction tool (BET) function. Eddy current and head motion artefact were corrected in FSL by aligning diffusion-weighted images to the first b0 image with an affine transformation with 12 degrees of freedom. The following four commonly used DTI measures were calculated using standard methods: fractional anisotropy (FA), mean diffusivity (MD), axial diffusivity (AD) and radial diffusivity (RD). ${ }^{23}$ The tractbased spatial statistics (TBSS) approach was used in the image analysis in the present study. ${ }^{24}$ This is a method developed to ameliorate the registration error at the boundary of narrow WM fibre bundles, a common source of error in voxel-based style analysis. Studies have shown that TBSS can effectively reduce the granularity and improve accuracy during normalisation. We followed standard TBSS analysis steps that have been summarised in many previous studies. ${ }^{25-27}$ A skeleton threshold of 0.2 was used in the present study.

\section{Neck ultrasound evaluation and collar fitting}

The neck collar device was made up of the following: an outer collar that consists of a thermoplastic elastomer (durometer 80 Shore A), an inner collar that consists of a thermoplastic elastomer (durometer 50 Shore A) and a stainless memory steel composite insert. Figure 1 shows the positioning of a collar compressing the jugular vein. The collar size for each individual was determined from the measured neck circumference, validated via spacing of the collar tips $\left(1.25-2.5^{\prime \prime}\right)$ and from visual evidence of ultrasound IJV dilation. At the initial fitting of the collar, a registered vascular technologist (JA) used ultrasound to ensure that the proper collar and IJV responses (eg, visual evidence of IJV dilation superior to collar) occurred as prescribed (figure 3).

A single sonographer performed all ultrasonography procedures and measurements, and the images and video clips were acquired using a LOGIQ e-unit (General Electric Inc, Fairfield, Connecticut, USA) with an ultrahigh frequency L8-18i-RS linear transducer. The study participants sat upright, facing forward during testing. IJV was identified in the transverse plane to verify normal anatomy. Each athlete was scanned for baseline, Valsalva (ie, bearing down against a closed glottis) and with the assigned collar on/off/on in sequence. A collar was then placed in proper position around the participant's neck. A $15 \mathrm{~s}$ video clip of the collar in proper position, opening away from the neck, and returning to its proper position, was obtained. The IJV metrics were measured at their largest transverse dimensions upon initial placement of the collar, when the collar pressure was removed, and again after replacing the collar back on the neck.

\section{Head impact surveillance}

Head impact was recorded using the GForce Tracker (GFT; GForce Tracker, Markham, Ontario, Canada) accelerometer device affixed to the inside of each football helmet. The accelerometers recorded linear acceleration and rotational velocity of the head (ie, 6 degrees of freedom) by directly measuring three axes of linear acceleration and three axes of angular acceleration. Prior to the initial exposure (ie, first practice), each accelerometer was calibrated according to device specifications and relative to the placement of the sensor in each helmet. Prior
Figure 3 Visual evidence of IJV dilation superior to omohyoid complex in response to Valsalva manoeuvre and with collar application. IJV, internal jugular vein.

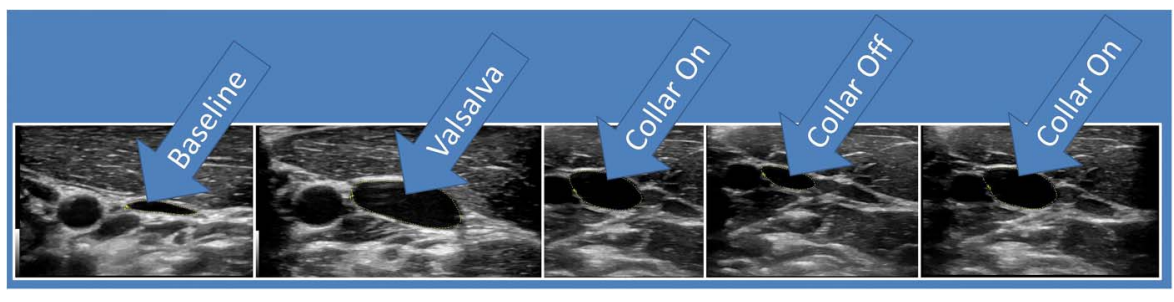


evaluation of GForce Tracker accelerometers indicates that they provide a suitable impact-monitoring device across multiple helmet styles with coefficients of determination reported at $r^{2}=0.82$ for peak linear acceleration. ${ }^{28}$ The accelerometers were programmed to record data above $10 \mathrm{~g}$, but only accelerations above $20 \mathrm{~g}$ threshold were used in the current investigation. Acceleration data were collected at $3000 \mathrm{~Hz}$. A more detailed description of accelerometer methods can be found in the online supplementary appendix 1 .

\section{Compliance of collar use}

Following the initial fitting, each athlete in the collar group received daily instruction on the proper usage of the collar. The collar usage was monitored by a study coordinator during routine visits, and recorded by the medical training staff in a daily log via custom software that tracked protocol compliance, attendance and any injury that occurred. These compliance logs were cross-referenced with accelerometer data daily to ensure data integrity for reported athlete exposures and collar compliance. Overall, the collar study participants (included in the reported per-protocol analysis) demonstrated $95.7 \%$ daily compliance (days of wear during impact practice or competition/ days possible) to collar usage as prescribed by the study protocol. It should be noted that the daily compliance collar usage was only $90.6 \%$ when evaluated by the intention-to-treat (ITT) methodology (see detailed results of ITT in the online supplementary appendix 1).

\section{Statistical analysis}

In the analysis of jugular vein dilation, a $4 \times 2$ repeated-measures analysis of variance (ANOVA) was computed to examine the per cent change difference in the dilation that occurred superior to the collar on the right and left sides for four different pairs of time points: (1) baseline to Valsalva, (2) baseline to collar on (time 1), (3) baseline to collar off and (4) baseline to collar on (time 2). The Bonferroni correction was used to correct for multiple follow-up tests.

The data indexing the total number of impacts were subjected to a Box-Cox natural $\log (\operatorname{Ln})$ transformation to alleviate extreme positive skewness observed in the data, ${ }^{29}$ while the total experienced g-forces were subjected to a simple Ln transformation due to moderate positive skewness. Separate independent t-tests were then conducted to compare the collar and CTRL groups for total number of impacts with various $\mathrm{g}$-force cut-offs $(>20,>50,>100 \mathrm{~g})$, total g-forces experienced $(>20 \mathrm{~g})$ and g-force per impact $(>20 \mathrm{~g})$. The frequency of impacts $>150$ and $>200 \mathrm{~g}$-force cut-off was too infrequent to conduct inferential analyses. All reported mean and SDs are the actual, non-transformed values.

In the neuroimaging analysis, the DTI measures (FA, $\mathrm{RD}, \mathrm{AD}$ and $\mathrm{MD}$ ) at a given voxel were first compared for group differences at baseline using an independent $t$-test. This was followed by a within-group, longitudinal change analysis between the two time points. For each participant, a difference map between the two time points was calculated for each DTI measure and used in a one-sample t-test to assess the longitudinal change for each group. Next, independent t-tests were used to determine whether the interaction between group and time was different by comparing the longitudinal change between the two groups. In each analysis step, the randomised function from FSL was used to generate a null distribution for comparison of the resulting t-test statistic for statistical significance. Permutations of 5000 and 2000 were used in each one-sample t-test and independent t-test, respectively. A multiple-comparison correction was achieved through the threshold-free cluster enhancement method $^{30}$ incorporated into the randomised software in FSL. We tested several variables, including time between last practice/ game to postseason MRI, the total number of impacts, the total g-force and the average g-force per impact, as covariates in the voxel-wise analysis of group differences of longitudinal DTI change. Each of these factors was initially tested individually for its potential confounding effect. We also tested the impact-related variables at several different g-force cut-off levels: $>20,>50$ and $>100 \mathrm{~g}$ as potential confounders. Our initial testing showed that none of these potential confounding variables had a significant effect in changing the contrast between the two groups in their preseason versus postseason DTI difference. Therefore, the final analyses of group differences presented in this study were conducted without including any of these variables. Based on the results from the WM areas with significant group differences of longitudinal change, we further explored whether the DTI change within these seemingly more vulnerable areas was associated with impact within the CTRL group. Before the correlation analysis was performed, the anatomical locations of the WM structures with significant group differences were determined by comparing with the John Hopkins University ICBM-DTI-81 WM labels atlas. ${ }^{31}{ }^{32}$ For each of the areas, the longitudinal change of DTI values was extracted for each participant in the CTRL group. A Pearson correlation analysis was performed between the change in DTI and each of the three impact-related variables.

Initially, all participants were assigned to either the collar or CTRL group (with 19 athletes in the CTRL group and 25 athletes in the collar group). However, two of the participants assigned to the treatment (collar) group refused to comply from the outset, and two were partially compliant with collar use during the competitive season. Therefore, all the neuroimaging analyses reported in the present study were performed based on the per-protocol design. This involved 21 athletes from the CTRL group and 21 athletes from the collar group. We also conducted analyses based on the ITT principle using the original allocation regardless of compliance status. As expected, similar but reduced effects were observed when the ITT analyses were used. The ITT analyses and results are described briefly below with the details included in the online supplementary appendix 1 for comparison.

\section{RESULTS}

\section{Responsiveness to collar}

Ultrasound evaluation confirmed that jugular vein size increased significantly above the level of the collar following collar placement, implying a backfill into the venous capacitance vessels of the cranium. While significant, the magnitude of change was less than that related to a quotidian physiologic Valsalva manoeuvre. A significant effect of time was indicated, independent of side, $\mathrm{F}(3,48)=12.88, \quad \mathrm{p}=0.002, \quad \eta^{2}=0.45 \quad$ (figure 4). Follow-up comparisons revealed that the per cent change for time point pair 1 (baseline to Valsalva) was significantly greater than all other time points $(\mathrm{p}<0.02)$. Time points 2 (baseline to collar on, time 1) and 4 (baseline to collar on, time 2) did not differ from each other, but both were significantly greater than time point 3 (baseline to collar off; $p<0.006$ ).

\section{Head impact surveillance}

There were no significant differences between the collar group and the CTRL group for any head impact measures (all $p>0.05$; table 1). As presented in figure 5 , the distribution of number of impacts at different $\mathrm{g}$-force indices was similar 


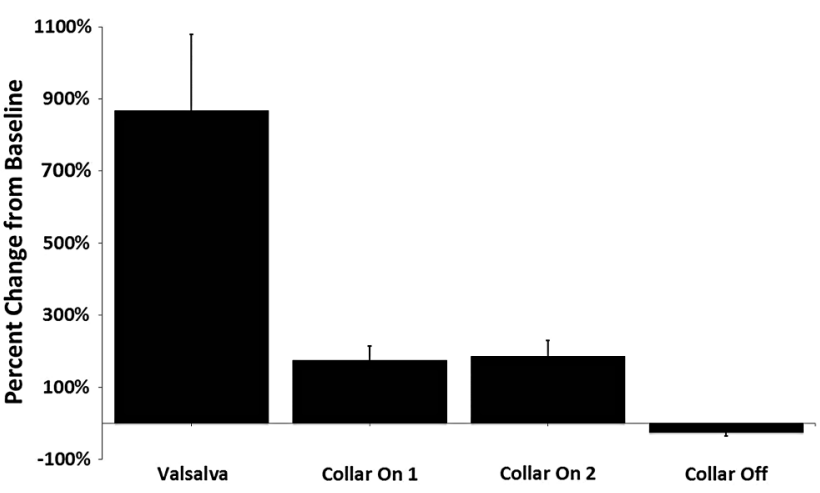

Figure 4 Relative change in IJV dilation from baseline measured superior to collar location for Valsalva and collar conditions. IJV, internal jugular vein.

between the two study groups (figure 6A, B). Table 1 presents descriptive values relative to each impact variable.

\section{Cross-sectional comparison of preseason DTI metrics between the CTRL group and the collar group}

At preseason, no significant differences were found in any of the four DTI measures in any WM region between the two groups.

\section{Longitudinal change and group difference in preseason to postseason DTI metrics}

Significant preseason to postseason decreases in MD, AD and $\mathrm{RD}$ (corrected $\mathrm{p}<0.05$ ) were found in extensive WM areas in the CTRL group (details below). No statistically significant preseason to postseason FA change was found in the CTRL group. No statistically significant longitudinal changes were found in any DTI measure in any WM region in the collar group $(\mathrm{p}>0.05)$.

Derived from the above within-group analyses, there is a consistent reduction of the diffusivity measures in the CTRL group, while the direction of change is more variable in the collar group (figure 7). For example, at an individual level, all 21 athletes in the CTRL group exhibited decreases in $\mathrm{AD}$ (percentage of reduction $=2.43 \pm 1.02 \%$; figure $8 \mathrm{~A}$ ). Within the same areas, 11 out of the 21 athletes in the collar group exhibited decreases in AD $(0.95 \pm 0.77 \%), 8$ out of the 21 athletes exhibited increases in AD $(1.22 \pm 0.68 \%)$ and 2 did not exhibit any change, with an overall

Table 1 Head impacts for per-protocol analysis

\begin{tabular}{llllll}
\hline \multicolumn{6}{l}{ (A) Average $( \pm S D)$ \# of hits experienced above impact level } \\
\hline \multicolumn{2}{c}{$\mathbf{2 0 ~ g}$} & $>\mathbf{5 0 ~ g}$ & $>100 \mathrm{~g}$ & $>150 \mathrm{~g}$ & $>\mathbf{2 0 0} \mathbf{~ g}$ \\
\hline No collar & $795.40 \pm 508.92$ & $145.85 \pm 109.98$ & $18.30 \pm 20.85$ & $1.80 \pm 2.04$ & $0.30 \pm 0.73$ \\
Collar & $896.43 \pm 600.03$ & $144.67 \pm 106.55$ & $14.38 \pm 11.29$ & $2.43 \pm 3.11$ & $0.43 \pm 1.17$
\end{tabular}

(B) Average $( \pm S D)$ of total $g$-forces experienced above impact level

\begin{tabular}{llll}
\hline & $>20 \mathrm{~g}$ & $>50 \mathrm{~g}$ & $>100 \mathrm{~g}$ \\
\hline No collar & $30065.43 \pm 19850.52$ & $10656.89 \pm 8549.30$ & $2377.46 \pm 2474.16$ \\
Collar & $32738.98 \pm 21873.88$ & $10486.55 \pm 7798.06$ & $1824.30 \pm 1499.10$ \\
\hline
\end{tabular}

(C) Average $\mathrm{g}$-force of hits above impact level

\begin{tabular}{llll}
\hline & $>\mathbf{2 0 ~ g}$ & $>50 \mathrm{~g}$ & $>100 \mathrm{~g}$ \\
\hline No collar & $38.15 \pm 4.24$ & $71.35 \pm 5.52$ & $124.20 \pm 7.96$ \\
Collar & $36.58 \pm 3.02$ & $72.37 \pm 3.95$ & $125.29 \pm 9.85$ \\
\hline
\end{tabular}
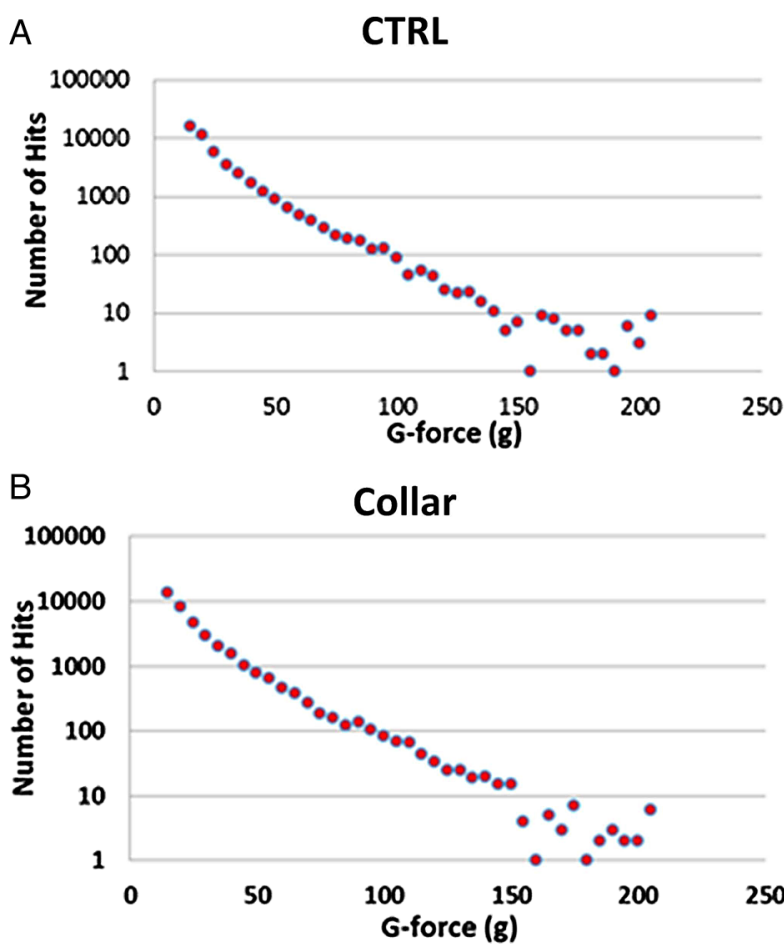

Figure 5 Histograms showing the distribution of number of impacts at different g-force. (A) No-collar (CTRL) group. (B) Collar group. CTRL, control.

average reduction of $\mathrm{AD}$ at the level of $0.03 \pm 1.27 \%$ or absolute change of $\mathrm{AD}(0.96 \pm 0.67 \%$; figure $8 \mathrm{~B})$.

Between-group analysis showed significantly larger preseason to postseason DTI changes in the CTRL group within the corpus callosum, the internal and external capsule, and a series of other WM regions (figure 9; corrected $\mathrm{p}<0.05$, see table 2 for location and volume of specific WM regions involved based on the $\mathrm{AD}$ measurement) when compared with the collar group (figure 10).

\section{Correlation between preseason to postseason DTI change in} the no-collar (CTRL) group and head impacts

Among the WM regions with significant group difference of $\mathrm{AD}$, the superior longitudinal fasciculus in the CTRL group was found to present significant correlation between the preseason and postseason decreases in diffusivity and impact burden. As shown in table 3 , the $\mathrm{AD}$ reduction in the superior longitudinal fasciculus in the CTRL group was significantly correlated with the number of hits and cumulative g-force (all hits or hits $\geq 20 \mathrm{~g}$ ) and marginally correlated with number of hits at the $\geq 50 \mathrm{~g}$ threshold. The MD reduction in superior longitudinal fasciculus in the CTRL group was mildly correlated with the number of hits and cumulative g-force (all hits or cut-off $>20 \mathrm{~g}$ ). In addition, we also found that the change of FA in posterior thalamic radiation was significantly correlated with the number of hits and with cumulative g-force (all hits or cut-off $>20 \mathrm{~g}$ ). No other WM region was found to have significant correlation between change in DTI measures and impact measures.

\section{Neuroimaging findings based on ITT protocol}

The results based on the ITT protocol are similar to the abovereported findings based on the per-protocol design. Briefly, based on the ITT design, we found significant preseason to postseason reduction in $\mathrm{MD}, \mathrm{AD}$ and $\mathrm{RD}$ in the CTRL group but 
Figure 6 (A) Graphical representation of average magnitude of head impacts (represented by height and colour) distributed about the sphere of the head for the CTRL (left, no-collar) and collar group (right). (B) Graphical representation of average frequency of head impacts (represented by height and colour) distributed about (left, no-collar) and collar group (right). CTRL, control. the sphere of the head for the CTRL
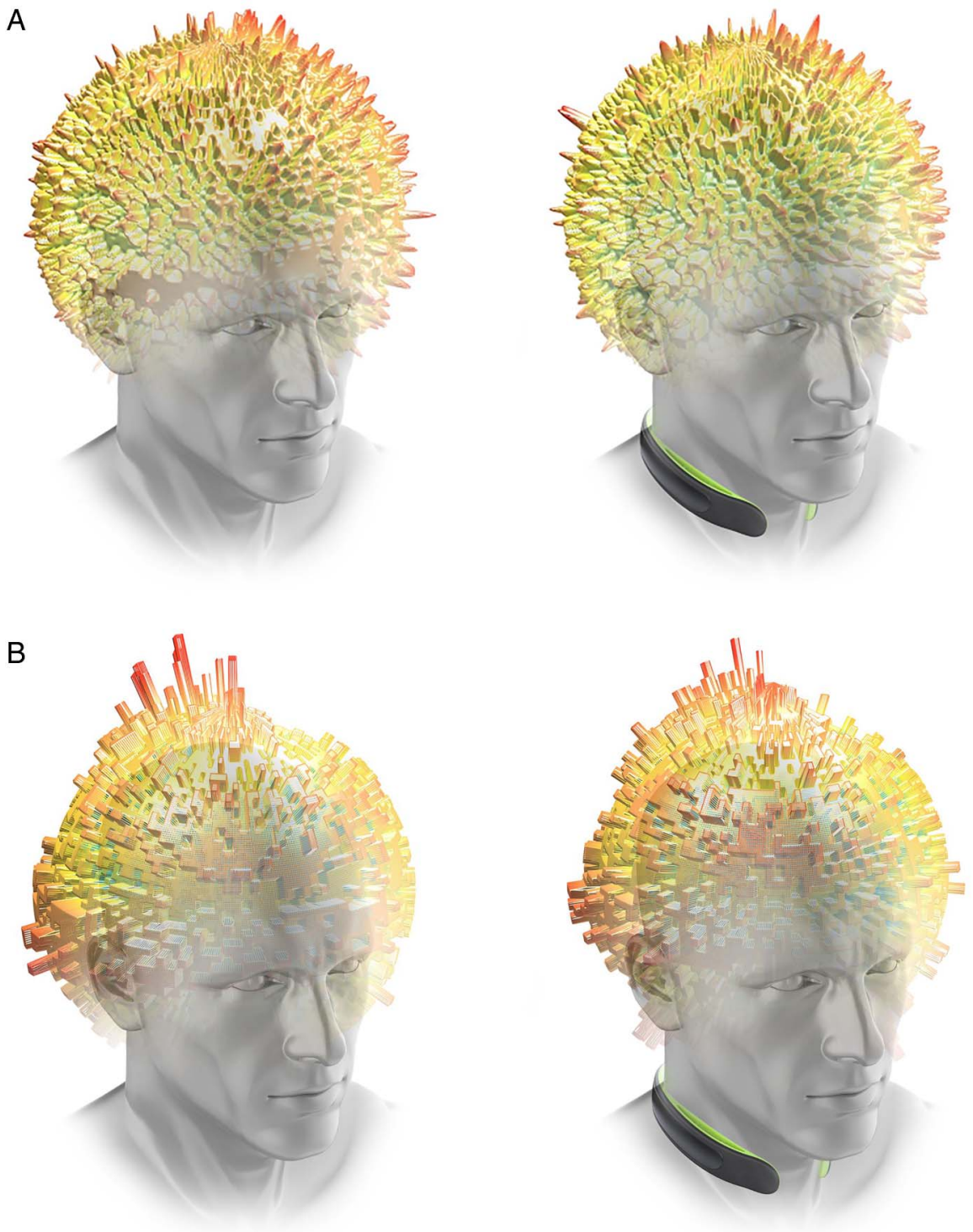

not in the collar group (see online supplementary figure S2). The group difference of preseason to postseason reduction in $\mathrm{MD}, \mathrm{AD}$ and RD remained statistically significant after using the collar group as the CTRL (see online supplementary figure S4). The WM regions with significant group difference of longitudinal diffusivity change were similar to the findings based on the per-protocol design (see online supplementary table S1). In the correlation analysis, the superior longitudinal fasciculus was found to present significant correlation between the AD reduction and the total number of hits. It should be noted that the number of WM regions with significant group difference and the number of WM regions with significant correlations in the ITT analysis were both smaller than that in the per-protocol design. This difference may be attributed to the lower compliance in the ITT design $(90.6 \%$ for ITT vs $95.7 \%$ for per protocol as reported in Method-Compliance of collar use). The ITT analysis which included two non-compliant athletes and the two partially compliant in the collar group weakened the homogeneity of the group analysis and, as expected, led to less significant findings. When compared to the formally presented per-protocol design, these data provide further support that the effect of collar wear to reduce changes in DTI was a valid outcome.

\section{DISCUSSION}

We examined the effect of a bilateral jugular vein compression collar device on the mitigation of brain microstructure changes as assessed by WM integrity in response to a seasonlong head impact exposure. The collar is a novel approach for the potential internal protection of the brain against repetitive head impact. As hypothesised, the wearing of the device was effective to prevent diffusion changes after a full season of repetitive head impacts in high school football athletes. Specifically, the CTRL group showed consistent and statistically significant reduction in diffusion coefficients-a change that has been reported frequently in $\mathrm{mTBI}^{33-37}$ and sports-related $\mathrm{TBI}^{22} \quad 38-44$ literature-contrasting with the absence of change in the football players who wore the collar. Combined with the association found between the decrease of diffusivity in the CTRL group and their impact burden, our study has generated initial evidence for a potential protective effect of the collar device. It supports the notion that the approach of protecting the brain internally via jugular vein compression may be a promising alternative, or at least a complementary tool, that helps to prevent and mitigate potential injury from repetitive head impact in competitive contact sports. 

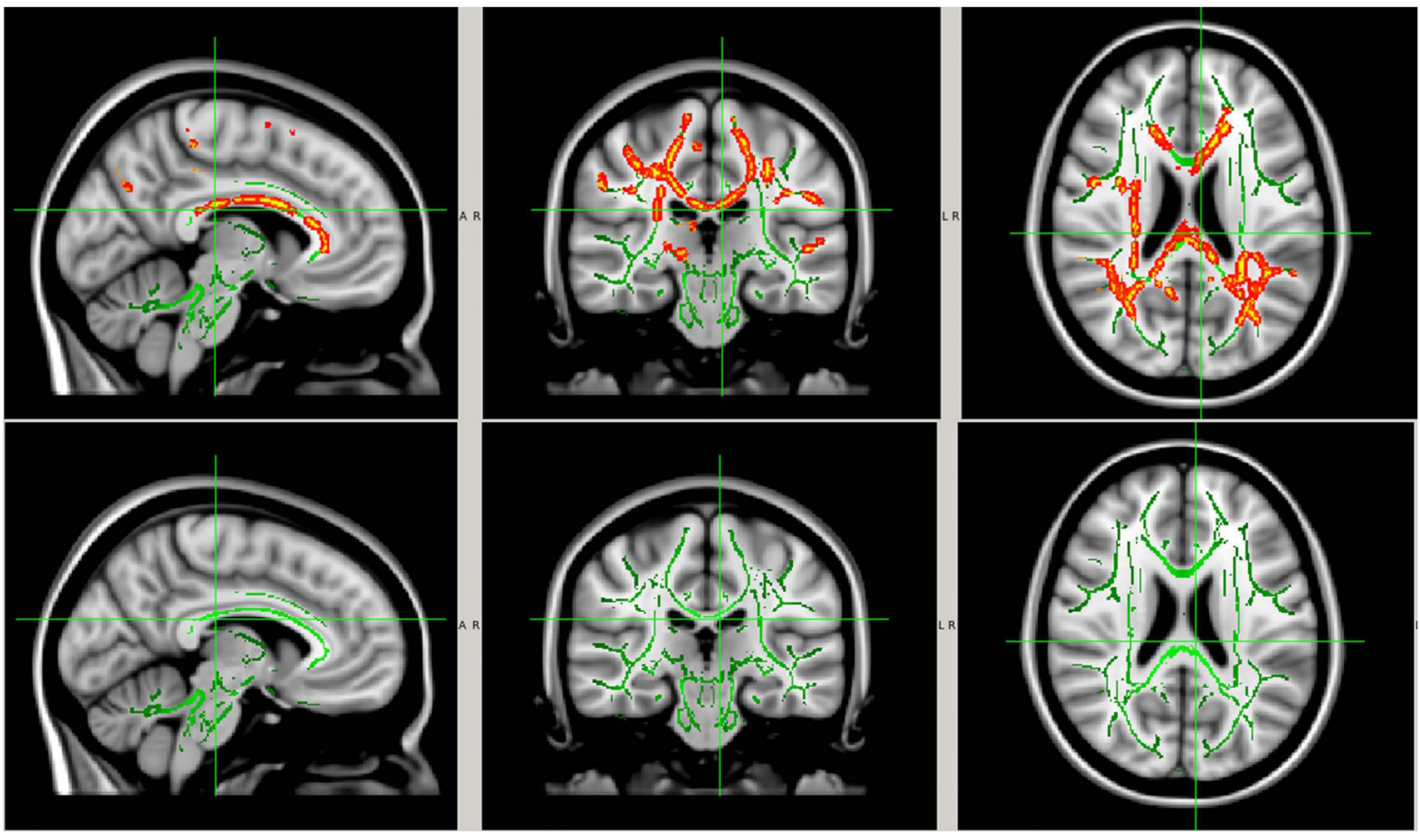

Figure 7 White matter regions with significant within-group AD reduction at postseason compared with preseason baseline. The significant regions (red-yellow regions, $p<0.05$, FWE corrected for multiple comparisons) were overlaid on to the white matter skeleton (green) and standard T1-weighted image in MNI 152 space (grey scale). The significant regions were filled in using tbss_fill in FSL to improve visualisation. Image orientation is in radiological convention. AD, axial diffusivity; CTRL, control; FSL, FMRIB Software Library; FWE, family-wise error rate; MNI, Montreal Neurological Institute. Top row: no-collar (CTRL) group; bottom row: collar group.

\section{Brain slosh and increased intracranial blood volume}

Slosh mechanisms have been primarily studied in their relation to fluid-filled containers. ${ }^{15}$ When an impact is sustained to a container filled with fluids (by extrapolation of the skull and brain), maximising volume within the container (ie, by reducing compliance of the venous sinuses and maximising intracranial blood and brain volume) reduces energy absorption. ${ }^{14}{ }^{15}$ It is likely that only a very small amount of intracranial blood volume change is necessary for this effect to occur. Accordingly, previous work has demonstrated that cerebral volume needs to rise only by $3-4 \mathrm{~mL}$ before concomitant pressure increases occur. ${ }^{45}$ Since the cerebral blood space is $\sim 150 \mathrm{~mL}$, we speculate that a $3 \%$ rise in intracranial volume will take up excess compliance and may provide protection from shear stress, cavitation and impact on the interior cranium. ${ }^{11} 12$ The degree of venous dilatation documented in this study with neck collar placement would not be expected to be dangerous and is likely less than the normal physiologic range noted with sneeze, cough and the Valsalva manoeuvre (ie, forced exhale against closed glottis; figures 3 and 4).

\section{Objectifying brain injury biomarkers in response to head} impacts

In contrast to the relatively subjective end point of concussion, DTI has been used as an objective imaging biomarker in quantifying WM structural changes in patients with mTBI and collision sport athletes during acute, semiacute and chronic stages after discrete head trauma or a season of repetitive head impacts. $^{22} 38-44$ WM integrity changes based on DTI have been correlated with postconcussive symptoms and cumulative risk-weighted exposure. ${ }^{40}$ Prior analyses of DTI in mTBI and sports-related TBI have shown changes of varying severity and direction, including abnormally lower FA and/or higher diffusivity $(\mathrm{MD}, \mathrm{AD}, \mathrm{RD})$ in patients, concussed athletes, athletes with a history of concussion, ${ }^{25274346}$ or decreased FA and/or increased diffusivity measures in athletes when tested for preseason to postseason changes. ${ }^{39} 424748$ However, other studies have shown changes in the opposite direction (ie, higher FA and/or lower diffusivity for $\mathrm{MD}, \mathrm{AD}$ and $\mathrm{RD}$ ) in acute $\mathrm{mTBI}^{33-37}$ and in collision sport athletes with either concussive or subconcussive

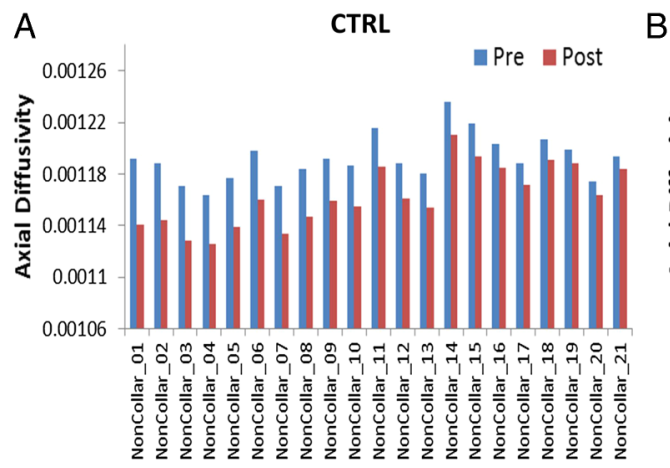

B

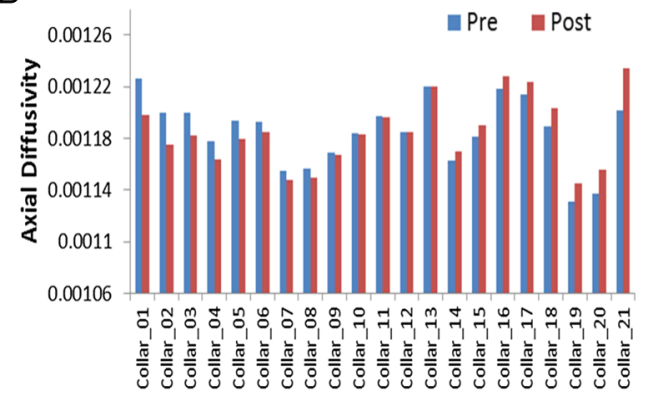

Figure 8 Bar plots of preseason and postseason $A D$ values from individual athletes. (A) No-collar (CTRL) group; (B) collar group. AD, axial diffusivity; CTRL, control. 

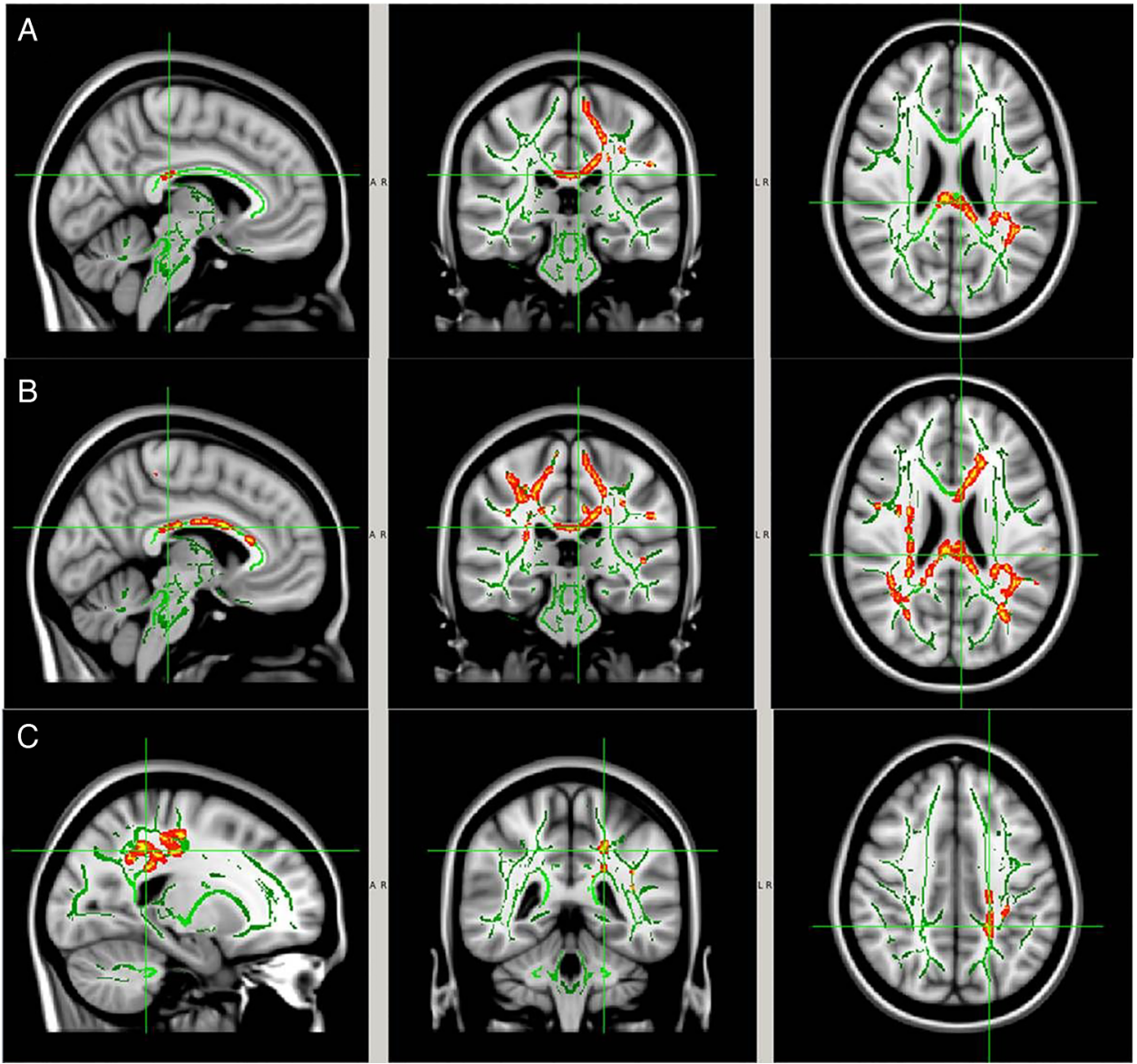

Figure 9 WM areas with significant between-group differences (CTRL vs collar), preseason versus postseason. (A) MD; (B) AD; and (C) RD. The significant regions (red-yellow regions, $\mathrm{p}<0.05$, FWE corrected for multiple comparisons) were overlaid on to the WM skeleton (green) and standard T1-weighted image in MNI 152 space (grey scale). The significant regions were filled in using tbss_fill in FSL to improve visualisation. Image orientation is in radiological convention. AD, axial diffusivity; FSL, FMRIB Software Library; FWE, family-wise error rate; MNI, Montreal Neurological Institute; MD, mean diffusivity; RD, radial diffusivity; WM, white matter.

impacts. $^{22} 38-44$ The inconsistency of these findings may be explained by the differences in timing of imaging, as well as differing severity and chronicity of brain injury.

In general, lower FA and higher $\mathrm{MD}, \mathrm{AD}$ and/or RD are often interpreted as damage to the myelin sheath and axonal membrane. Conversely, higher FA and lower MD, $\mathrm{AD}$ and/or RD are often attributed to extracellular space compression, cytotoxic oedema (axonal swelling) or inflammation. ${ }^{49} 50$ In the present study, no significant change in FA was noted: the primary findings were significant decreases in WM diffusivity at postseason in the CTRL group. The areas affected included the corpus callosum, anterior and posterior internal capsule, corona radiata, posterior thalamic radiation, external capsule, cingulum and superior longitudinal fasciculus, which are consistent with previous studies of TBI and head impacts. ${ }^{22} 252733-4446-50$ Previously, a prospective randomised controlled trial was performed in hockey players to evaluate the effects of external jugular compression applied during head impact exposure on longitudinal changes in brain neuroanatomical and neurophysiological biomarkers. ${ }^{19}$ Similar to the current results, athletes not wearing the collar and exposed to head impacts showed a disruption of WM microstructure, including mean diffusivity and radial diffusivity. Hockey players who wore the compressive collar did not show a significant change in either MD or RD despite similar accumulated linear accelerations from head impacts. In addition to these anatomical findings, electrophysiological network analysis demonstrated concomitant changes in brain network dynamics in the no-collar
Table 2 The WM regions corresponding to the brain regions with significant preseason versus postseason changes (figure 10) were determined using John Hopkins University's WM atlas

\begin{tabular}{|c|c|c|}
\hline & $\begin{array}{l}\text { Volume } \\
\left(\mathrm{mm}^{3}\right)\end{array}$ & WM region located \\
\hline Region 01 & 173 & Genu of corpus callosum \\
\hline Region 02 & 960 & Body of corpus callosum \\
\hline Region 03 & 384 & Splenium of corpus callosum \\
\hline Region 04 & 3 & Anterior limb of internal capsule $R$ \\
\hline Region 05 & 40 & Posterior limb of internal capsule $\mathrm{R}$ \\
\hline Region 06 & 84 & Retrolenticular part of internal capsule $\mathrm{R}$ \\
\hline Region 07 & 2 & Retrolenticular part of internal capsule $\mathrm{L}$ \\
\hline Region 08 & 216 & Anterior corona radiata $\mathrm{L}$ \\
\hline Region 09 & 490 & Superior corona radiata $\mathrm{R}$ \\
\hline Region 10 & 77 & Superior corona radiata $\mathrm{L}$ \\
\hline Region 11 & 252 & Posterior corona radiata $\mathrm{R}$ \\
\hline Region 12 & 266 & Posterior corona radiata $\mathrm{L}$ \\
\hline Region 13 & 76 & Posterior thalamic radiation (include optic radiation) $R$ \\
\hline Region 14 & 18 & Posterior thalamic radiation (include optic radiation) L \\
\hline Region 15 & 22 & External capsule $\mathrm{R}$ \\
\hline Region 16 & 42 & Cingulum (cingulate gyrus) L \\
\hline Region 17 & 365 & Superior longitudinal fasciculus $R$ \\
\hline Region 18 & 197 & Superior longitudinal fasciculus $L$ \\
\hline Region 19 & 2 & Tapetum R \\
\hline
\end{tabular}

WM, white matter. 

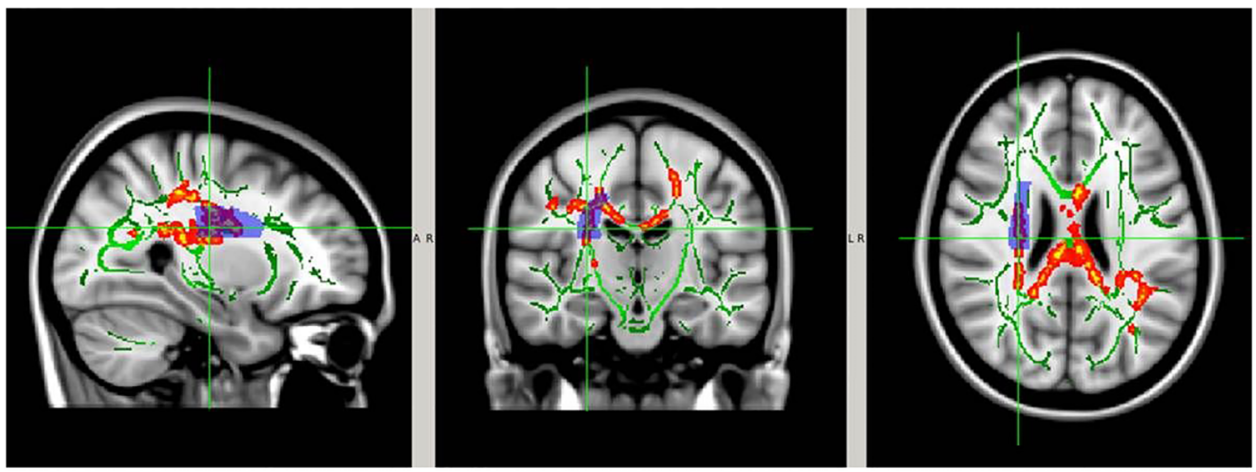

Figure 10 The right superior corona radiata (light blue shadow area as determined by John Hopkins University s WM atlas) overlaid on the white matter regions with significant preseason versus postseason AD change (red-yellow area, $p<0.05$, FWE corrected, filled in using tbss_fill in FSL to improve visualisation) in the CTRL group, the white matter skeleton (green) and the standard T1-weighted image in MNI 152 space. AD, axial diffusivity; CTRL, control; FSL, FMRIB Software Library; FWE, family-wise error rate; MNI, Montreal Neurological Institute.

(CTRL) group only. Interestingly, the changes in DTI outcome (RD) were directly correlated with altered brain network dynamics $(r=0.76)$ in this cohort. ${ }^{19}$

Repetitive, subconcussive brain injury may result in a mix of acute, subacute and chronic cellular changes over a season of contact sports that can produce complex changes in the brain's water diffusion. ${ }^{20}$ Assessing DTI metrics at a single time point evaluates the cumulative effects of brain injury and repair present at that time point. ${ }^{22}$ The findings in the present study lend support to this notion. Specifically, in a longitudinal study of DTI changes in response to repetitive subconcussive impacts over a season, postseason imaging should be considered as a reflection of the changes in brain microstructure that resulted from repetitive subconcussive hits accumulated over the entire season.

\section{Head impact exposure in American football}

In the present study, the number of sessions in which impacts were recorded was slightly higher for the collar group than was reported in the CTRL group (72 vs 60). Other studies of football-related impacts have evaluated seasons of similar duration (60-68 practices and games). ${ }^{51}$ The average g-force per impact reported over the course of the season in the present study (36.6-37.7 g) was higher than has been reported in most prior studies of contact sports (ranging from 20.9 to $32.0 \mathrm{~g}),{ }^{51-56}$ which may be attributed to the higher threshold for recording valid impacts in the current study $(>20 \mathrm{~g})$ than the other reports $(>10-15 \mathrm{~g}) .^{51-56}$ The number of average hits per player reported in the current investigation (ranging from 794 to 901) was also higher than has been reported in most previous reports as well. ${ }^{51-56}$ For example, Broglio et $a l^{51}$ reported an average of $\sim 549$ impacts per player $(>15 \mathrm{~g})$ in a season and an average of 774 impacts per player $(>14.4 \mathrm{~g})$ in the same team in another season. ${ }^{52}$ Similarly, Schnebel et al ${ }^{57}$ reported an average of 520 impacts per player $(>10 \mathrm{~g})$. Comparably, at the
Division I Football Bowl Subdivision collegiate level, the average number of impacts per player over the course of a season has varied widely, ranging from 171 to $1354 . .^{53-57}$ Our results showed no significant effect of impact per player or any other impact-related variable in any of the covariate analyses on the potential collar effects on reduced change in DTI. The current data are generalisable in relative head impact exposure (magnitude and quantity) to high school and collegiate football players over a single season.

\section{Limitations}

In the present study, the similar impact exposure between the two study groups strengthens the conclusion that the significantly lower levels of DTI alterations in the collar group are attributed to the intervention. However, despite best efforts for control, the changes reported could be potentially explained by other factors such as physiologic responses to other stresses in addition to trauma, developmental changes of the immature brain, genetics and/or other susceptibilities. The utilisation of a controlled longitudinal study design can mitigate, but does not eliminate, the risk of these potential confounding variables. Regardless, the cumulative results of the current investigation represent a substantive and important finding in the field of sports injury prevention. The exact pathologic substrate and clinical significance of the diffusion changes identified in this study are unknown. The diffusion changes and locations identified in the CTRL group are consistent with those identified in prior studies of severe TBI (sTBI), lending support to the premise that they are traumatic in origin. In addition, the current investigation was focused on the assessment of objective biomarkers that are associated with brain injury, and the study design was not appropriate to evaluate the subjective and often nebulous outcome of clinically diagnosed concussion. The authors acknowledge future large-scale epidemiological clinical trials are needed to determine the potential of the investigated

Table 3 Association between change in DTI in the CTRL group and the impact-related measures (correlation coefficient/p value)

\begin{tabular}{lrrrrrr}
\hline & Hits & Hits $(>20 \mathrm{~g})$ & Hits $(>50 \mathrm{~g})$ & Cumug & Cumug $(>20 \mathrm{~g})$ & Cumug $(>50 \mathrm{~g})$ \\
\hline$\Delta \mathrm{AD}$ in SLF & $0.49 / 0.012$ & $0.46 / 0.017$ & $0.34 / 0.066$ & $0.47 / 0.015$ & $0.44 / 0.022$ & $0.29 / \mathrm{NS}$ \\
$\Delta \mathrm{MD}$ in SLF & $0.49 / 0.012$ & $0.46 / 0.019$ & $0.27 / \mathrm{NS}$ & $0.46 / 0.019$ & $0.42 / 0.057$ & $0.20 / \mathrm{NS}$ \\
$\Delta$ FA in PTR & $-0.58 / 0.003$ & $-0.41 / 0.032$ & $-0.22 / \mathrm{NS}$ & $-0.42 / 0.030$ & $-0.37 / 0.049$ & $-0.21 / \mathrm{NS}$ \\
\hline
\end{tabular}

Correlation coefficient/p value. All statistics were tested using one-tailed Pearson correlation at $p<0.05$.

$A D$, axial diffusivity; CTRL, control; DTI, diffusion tensor imaging; FA, fractional anisotropy; MD, mean diffusivity; NS, not significant; PTR, posterior thalamic radiation; SLF: superior longitudinal fasciculus. 
collar device for mitigating concussion incidence. Finally, future research is warranted to understand and document the intracranial effects of jugular venous compression, to assess for any long-term effects of playing sport with jugular venous compression and presumably increased cerebral blood volume, as well as to validate its mitigating effects on brain injury from head impact exposure.

\section{CONCLUSION}

The current investigation tested a neck collar device that produces physiologic distension of the superior jugular veins to encourage cerebral venous engorgement to ameliorate the changes in brain microstructure associated with a season of contact sport. These prospective longitudinal data using an internal in vivo approach indicate that it may be possible to protect the brain from 'inside the skull' from sports-related head impacts.

\section{What are the findings?}

- Analyses of brain injury biomarkers and head impacts in response to the season-long application of a jugular compression collar indicate a consistent reduction in altered white matter diffusivity parameters.

- These results represent prospective, longitudinal and objective data demonstrating a potential approach to protect the brain from changes sustained within a competitive football season, as evidenced by brain microstructure integrity.

\section{How might it impact on clinical practice in the future?}

- These prospective longitudinal data using an internal in vivo approach indicate that it may be possible to protect the brain from 'inside the skull' from sports-related head impacts.

- The novelty of this device, as well as the tolerance and general compliance, may help drive future efforts to prevent sports-related brain injury.

\section{Author affiliations}

${ }^{1}$ Division of Sports Medicine, Cincinnati Children's Hospital Medical Center, Cincinnati, Ohio, USA

${ }^{2}$ Division of Sports Medicine, The SPORT Center, Cincinnati Children's Hospital Medical Center, Cincinnati, Ohio, USA

${ }^{3}$ Department of Pediatrics, University of Cincinnati, College of Medicine, Cincinnati, Ohio, USA

${ }^{4}$ Department of Orthopaedics, University of Pennsylvania, Philadelphia, Pennsylvania, USA

${ }^{5}$ The Micheli Center for Sports Injury Prevention, Waltham, Massachusetts, USA

${ }^{6}$ Department of Orthopaedic Surgery, University of Cincinnati, Cincinnati, Ohio, USA

${ }^{7}$ Pediatric Neuroimaging Research Consortium, Cincinnati Children's Hospital Medical Center, Cincinnati, Ohio, USA

${ }^{8}$ College of Medicine, University of Cincinnati, Cincinnati, Ohio, USA

${ }^{9}$ Division of Health Sciences, Department of Athletic Training, Mount St. Joseph University, Cincinnati, Ohio, USA

${ }^{10}$ Rocky Mountain University of Health Professions, Provo, Utah, USA

${ }^{11}$ Division of Radiology, Cincinnati Children's Hospital Medical Center, Cincinnati, Ohio, USA

${ }^{12}$ Department of Psychology, Center for Cognition, Action and Perception, University of Cincinnati, Cincinnati, Ohio, USA

${ }^{13}$ College of Medicine, University of Cincinnati, Cincinnati, Ohio, USA
${ }^{14}$ Division of Neurology, Cincinnati Children's Hospital Medical Center, Cincinnati, Ohio, USA

${ }^{15}$ Division of Biostatistics and Epidemiology, Cincinnati Children's Hospital Medical Center, Cincinnati, Ohio, USA

Twitter Follow Gregory Myer at @gregmyer11

Acknowledgements The authors would like to thank Steve Specht, John Rodenberg, John Sullivan, Michael Asbeck, Tom Gamble, Michael Gordon, Craig Lindsay, Ken Rushford and Carlee Shafer for their support and assistance to conduct this study. Thank you to the football parents and players. We appreciate their patience with the testing, scheduling, follow-ups and equipment additions. The authors would like to thank Lacey Haas, Brynne Williams and Matt Lanier in the Imaging Research Center. Their support made this study possible. Funding for this study was provided by the Heidt Family Foundation, Robert S. Heidt, Sr.Wellington Foundation and Q30 Sports Sciences, LLC. Q30 Sports Sciences has financial interests in the development of the Q-Collar.

Contributors GDM contributed to the conception and design, acquisition of data, analysis and interpretation of data, drafting of the manuscript, obtaining funding and supervision. WY contributed to the conception and design, analysis and interpretation of data, critical revision of the manuscript for important intellectual content, and analysis and interpretation of data. KDBF contributed to the acquisition of data, drafting of the manuscript, and administrative, technical or material support. ST contributed to the acquisition of data, drafting of the manuscript, and administrative, technical, or material support. DS contributed to the conception and design, drafting of the manuscript and material support. $\mathrm{JL}$ contributed to the conception and design, critical revision of the manuscript for important intellectual content, and analysis and interpretation of data. AWK contributed to the conception and design, critical revision of the manuscript for important intellectual content, and analysis and interpretation of data. CD contributed to the acquisition of data, drafting of the manuscript, and administrative, technical or material support. JA contributed to the acquisition of data, drafting of the manuscript, and administrative, technical or material support. PJG contributed to the analysis and interpretation of data, critical revision of the manuscript. KK, DKS and DB contributed to the acquisition of data, drafting of the manuscript, and administrative, technical or material support. DK contributed to the conception and design, critical revision of the manuscript for important intellectual content, and analysis and interpretation of data. MA contributed to the analysis and interpretation of data, critical revision of the manuscript for important intellectual content and statistical analysis.

Funding Q30 Innovations (Funding Proposal: FP00009108).

Competing interests David Smith is the inventor of the Q-Collar approach and has financial interest in the results of the current research.

Patient consent Obtained.

Ethics approval Cincinnati Children's Hospital Medical Center Institutional Review Board.

Provenance and peer review Not commissioned; externally peer reviewed.

Open Access This is an Open Access article distributed in accordance with the Creative Commons Attribution Non Commercial (CC BY-NC 4.0) license, which permits others to distribute, remix, adapt, build upon this work non-commercially, and license their derivative works on different terms, provided the original work is properly cited and the use is non-commercial. See: http://creativecommons.org/ licenses/by-nc/4.0/

\section{REFERENCES}

1 Daneshvar DH, Nowinski CJ, McKee AC, et al. The epidemiology of sport-related concussion. Clin Sports Med 2011;30:1-17, vii.

2 Fridman L, Fraser-Thomas JL, McFaull SR, et al. Epidemiology of sports-related injuries in children and youth presenting to Canadian emergency departments from 2007-2010. BMC Sports Sci Med Rehabil 2013;5:30

3 Schneider DK, Grandhi RK, Bansal P, et al. Current state of concussion prevention strategies: a systematic review and meta-analysis of prospective, controlled studies Br J Sports Med Published Online First: 1 Jun 2016 doi:10.1136/bjsports-2015095645

4 Barbic D, Pater J, Brison RJ. Comparison of mouth guard designs and concussion prevention in contact sports: a multicenter randomized controlled trial. Clin I Sport Med 2005;15:294-8.

5 Kemp SP, Hudson Z, Brooks JH, et al. The epidemiology of head injuries in English professional rugby union. Clin J Sport Med 2008;18:227-34.

6 Marshall SW, Loomis DP, Waller AE, et al. Evaluation of protective equipment for prevention of injuries in rugby union. Int I Epidemiol 2005;34:113-18.

7 McGuine T, Brooks A, Hetzel S, et al. The association of the type of football helmet and mouth guard with the incidence of sport related concussion in High School football players. Orthop J Sports Med 2013;1. 
8 Winters J, DeMont R. Role of mouthguards in reducing mild traumatic brain injury/ concussion incidence in high school football athletes. Gen Dent 2014;62:34-8.

9 Lloyd J, Conidi F. Brain injury in sports. J neurosurg 2016;124:667-74.

10 Benson BW, Hamilton GM, Meeuwisse WH, et al. Is protective equipment useful in preventing concussion? A systematic review of the literature. Br J Sports Med 2009;43(Suppl 1):i56-67.

11 Smith DW, Myer GD, Currie DW, et al. Altitude modulates concussion incidence: implications for optimizing brain compliance to prevent brain injury in athletes. Orthop J Sports Med 2013;1:2325967113511588.

12 Myer GD, Smith D, Barber Foss KD, et al. Rates of concussion are lower in National Football League games played at higher altitudes. J Orthop Sports Phys Ther 2014:44:164-72.

13 Gilland 0 , Chin F, Anderson WB, et al. A cinemyelographic study of cerebrospinal fluid dynamics. Am J Roentgenol Radium Ther Nucl Med 1969;106:369-75.

14 Smith DW, Bailes JE, Fisher JA, et al. Internal jugular vein compression mitigates traumatic axonal injury in a rat model by reducing the intracranial slosh effect. Neurosurgery 2012;70:740-6.

15 Turner RC, Naser ZJ, Bailes JE, et al. Effect of slosh mitigation on histologic markers of traumatic brain injury: laboratory investigation. J Neurosurg 2012;117:1110-18.

16 Leach JL, Smith DW, Myer GD. Mild Neck Compression Alters Intracranial Venous Sinus Volume: Implications for a Novel Neuroprotective Effect in Concussion. American Society of Neuroradiology Annual Meeting:2013.

17 Mongan P, Sulpicio S, Sloan TB, et al. A practical approach to neuroanesthesia. Wolters Kluwer Health, 2013

18 Hatt A, Cheng S, Tan K, et al. MR elastography can be used to measure brain stiffness changes as a result of altered cranial venous drainage during jugular compression. AJNR Am J Neuroradiol 2015:36:1971-7.

19 Myer GD, Yuan W, Barber Foss KD, et al. The effects of external jugular compression applied during head impact exposure on longitudinal changes in brain neuroanatomical and neurophysiological biomarkers: a preliminary investigation. Front Neurol Section Neurotrauma 2016:7:74-88.

20 Hulkower MB, Poliak DB, Rosenbaum SB, et al. A decade of DTI in traumatic brain injury: 10 years and 100 articles later. AJNR Am J Neuroradiol 2013:34:2064-74.

21 Virji-Babul N, Borich MR, Makan N, et al. Diffusion tensor imaging of sports-related concussion in adolescents. Pediatr Neurol 2013:48:24-9.

22 Bazarian JJ, Zhu T, Zhong J, et al. Persistent, long-term cerebral white matter changes after sports-related repetitive head impacts. PLOS ONE 2014;9:e94734.

23 Basser PJ, Pierpaoli C. A simplified method to measure the diffusion tensor from seven MR images. Magn Reson Med 1998:39:928-34.

24 Smith SM, Jenkinson M, Johansen-Berg $\mathrm{H}$, et al. Tract-based spatial statistics: voxelwise analysis of multi-subject diffusion data. Neuroimage 2006;31:1487-505.

25 Fakhran S, Yaeger $\mathrm{K}$, Collins M, et al. Sex differences in white matter abnormalities after mild traumatic brain injury: localization and correlation with outcome. Radiology 2014;272:815-23.

26 Sasaki T, Pasternak O, Mayinger M, et al. Hockey Concussion Education Project, Part 3. White matter microstructure in ice hockey players with a history of concussion: a diffusion tensor imaging study. J Neurosurg 2014;120:882-90.

27 Cubon VA, Putukian $M$, Boyer $C$, et al. A diffusion tensor imaging study on the white matter skeleton in individuals with sports-related concussion. J Neurotrauma 2011:28:189-201.

28 Campbell KR, Warnica MJ, Levine IC, et al. Laboratory evaluation of the gForce tracker, a head impact kinematic measuring device for use in football helmets. Ann Biomed Eng 2016:44:1246-56.

29 Box GE, Cox DR. An analysis of transformations. J R Stat Soc Ser $B$ (Methodological) 1964:211-52.

30 Smith SM, Nichols TE. Threshold-free cluster enhancement: addressing problems of smoothing, threshold dependence and localisation in cluster inference. Neuroimage 2009;44:83-98.

31 Mori $\mathrm{S}$, Oishi $\mathrm{K}$, Jiang $\mathrm{H}$, et al. Stereotaxic white matter Atlas based on diffusion tensor imaging in an ICBM template. Neuroimage 2008;40:570-82.

32 Mori S, Wakana S, Van Zijl PC, et al. MRI Atlas of human white matter. Am Soc Neuroradiology 2005

33 Mayer AR, Ling J, Mannell MV, et al. A prospective diffusion tensor imaging study in mild traumatic brain injury. Neurology 2010;74:643-50.
34 Wilde EA, McCauley SR, Hunter JV, et al. Diffusion tensor imaging of acute mild traumatic brain injury in adolescents. Neurology 2008;70:948-55.

35 Chu Z, Wilde EA, Hunter JV, et al. Voxel-based analysis of diffusion tensor imaging in mild traumatic brain injury in adolescents. AJNR Am J Neuroradiol 2010;31:340-6

36 Wilde EA, McCauley SR, Barnes A, et al. Serial measurement of memory and diffusion tensor imaging changes within the first week following uncomplicated mild traumatic brain injury. Brain Imaging Behav 2012;6:319-28.

37 Babcock L, Yuan W, Leach J, et al. White matter alterations in youth with acute mild traumatic brain injury. J Pediatr Rehabil Med 2015;8:285-96.

38 Chamard $E$, Lefebvre $G$, Lassonde $M$, et al. Long-Term Abnormalities in the Corpus Callosum of Female Concussed Athletes. J Neurotrauma 2015. ahead of print. doi:10.1089/neu.2015.3948

39 Bartnik-Olson BL, Holshouser B, Wang $\mathrm{H}$, et al. Impaired neurovascular unit function contributes to persistent symptoms after concussion: a pilot study. J Neurotrauma 2014;31:1497-506.

40 Davenport EM, Whitlow CT, Urban JE, et al. Abnormal white matter integrity related to head impact exposure in a season of high school varsity football. J Neurotrauma 2014;31:1617-24.

41 Bazarian JJ, Zhu T, Blyth B, et al. Subject-specific changes in brain white matter on diffusion tensor imaging after sports-related concussion. Magn Reson Imaging 2012:30:171-80.

42 McAllister TW, Ford JC, Ji S, et al. Maximum principal strain and strain rate associated with concussion diagnosis correlates with changes in corpus callosum white matter indices. Ann Biomed Eng 2012;40:127-40.

43 Henry LC, Tremblay J, Tremblay S, et al. Acute and chronic changes in diffusivity measures after sports concussion. J Neurotrauma 2011;28: 2049-59.

44 Zhang K, Johnson B, Pennell D, et al. Are functional deficits in concussed individuals consistent with white matter structural alterations: combined FMRI \& DTI study. Exp Brain Res 2010;204:57-70.

45 Löfgren J, Zwetnow NN. Cranial and spinal components of the cerebrospinal fluid pressure-volume curve. Acta Neurol Scand 1973;49:575-85.

46 Chamard E, Lassonde M, Henry L, et al. Neurometabolic and microstructural alterations following a sports-related concussion in female athletes. Brain Inj 2013;27:1038-46.

47 Murugavel $\mathrm{M}$, Cubon V, Putukian $\mathrm{M}$, et al. A longitudinal diffusion tensor imaging study assessing white matter fiber tracts after sports-related concussion. J Neurotrauma 2014:31:1860-71.

48 Koerte IK, Kaufmann D, Hartl E, et al. A prospective study of physician-observed concussion during a varsity university hockey season: white matter integrity in ice hockey players. Part 3 of 4. Neurosurg Focus 2012;33(6):E3:1-7.

49 Barzó P, Marmarou A, Fatouros P, et al. Contribution of vasogenic and cellular edema to traumatic brain swelling measured by diffusion-weighted imaging. J Neurosurg 1997;87:900-7

50 Niogi SN, Mukherjee P. Diffusion tensor imaging of mild traumatic brain injury. J Head Trauma Rehabil 2010;25:241-55

51 Broglio SP, Sosnoff JJ, Shin S, et al. Head impacts during high school football: a biomechanical assessment. J Ath/ Train 2009;44:342-9.

52 Broglio SP, Martini D, Kasper L, et al. Estimation of head impact exposure in high school football: implications for regulating contact practices. Am J Sports Med 2013:41:2877-84

53 Brolinson PG, Manoogian S, McNeely D, et al. Analysis of linear head accelerations from collegiate football impacts. Curr Sports Med Rep 2006;5: 23-8.

54 Duma SM, Manoogian SJ, Bussone WR, et al. Analysis of real-time head accelerations in collegiate football players. Clin I Sport Med 2005; 15:3-8

55 Mihalik JP, Bell DR, Marshall SW, et al. Measurement of head impacts in collegiate football players: an investigation of positional and event-type differences. Neurosurgery 2007;61:1229-35.

56 Rowson S, Brolinson G, Goforth $M$, et al. Linear and angular head acceleration measurements in collegiate football. J Biomech Eng 2009;131:061016.

57 Schnebel B, Gwin JT, Anderson S, et al. In vivo study of head impacts in football: a comparison of National Collegiate Athletic Association Division I versus high school impacts. Neurosurgery 2007:60:490-5; discussion 95-6. 